\title{
Analitik fonksiyonlar teorisinde Pascal tipi dağılım serileri ile uygulamaları üzerine bir çalışma
}

A study on the Pascal type distribution series with applications in analytic function theory

\section{Şahsene ALTINKAYA*1,2a}

${ }^{1}$ Beykent Üniversitesi, Fen Edebiyat Fakültesi, Matematik Bölümü, 34500, İstanbul

${ }^{2}$ Bursa Uludağ Üniversitesi, Fen Edebiyat Fakültesi, Matematik Bölümü, 16059, Bursa

• Geliş tarihi / Received: 28.03.2020 •Düzeltilerek geliş tarihi / Received in revised form: 30.10 .2020 • Kabul tarihi / Accepted: 30.12 .2020

$\ddot{O} z$

Bu güncel çalışmada,

$P(X=n)=\left(\begin{array}{c}n+k-1 \\ k-1\end{array}\right) p^{n}(1-p)^{k} \quad(n \in\{0,1,2,3, \ldots\})$

formundaki Pascal tipli dağılım serisi kullanılarak iki kuvvet serisi $P_{\sigma}(k, p, z)$ ve $P(k, p, z)$ tanımlanmıştır. Daha sonra bu serilerin $\operatorname{USTN}(\beta)$ analitik fonksiyon sınıfına ait olması için gerekli şartlar geliştirilmiştir.

Anahtar kelimeler: Analitik fonksiyonlar, Dağılım serileri, Yıldızıl fonksiyonlar

\section{Abstract}

In this current study, by using the Pascal type distribution series of the form

$$
P(X=n)=\left(\begin{array}{c}
n+k-1 \\
k-1
\end{array}\right) p^{n}(1-p)^{k} \quad(n \in\{0,1,2,3, \ldots\})
$$

we define two power series $P_{\sigma}(k, p, z)$ and $P(k, p, z)$. Afterwards, we develop sufficient conditions for these series to be in the class $\operatorname{USTN}(\beta)$ of analytic functions.

Keywords: Analytic functions, Distribution series, Starlike functions

\footnotetext{
*a Şahsene ALTINKAYA; sahsenealtinkaya@beykent.edu.tr, Tel: 444 1997, orcid.org/0000-0002-7950-8450
} 


\section{Giriş}

$\mathbb{C}$ kompleks düzlem olmak üzere $U=\{z: z \in$ $\mathbb{C}$ ve $|z|<1\}$ açık birim disk olarak tanımlanır. $D$ kompleks düzlemde bir bölge olmak üzere $f: D \rightarrow$ $\mathbb{C}$ fonksiyonu birebir ise $f$ fonksiyonuna yalınkat fonksiyon denir. $f: D \rightarrow \mathbb{C}$ sürekli dönüşümü için bir $z_{0} \in D$ noktasından geçen ve aralarında $\alpha$ açısı bulunan herhangi iki düzgün $\gamma_{1}$ ve $\gamma_{2}$ eğrilerinin; $f\left(\gamma_{1}\right)$ ve $f\left(\gamma_{2}\right)$ resim eğrilerinin de $w_{0}=f\left(z_{0}\right)$ noktasında aralarında yön ve büyüklük bakımından $\alpha$ açısı varsa, $f$ fonksiyonuna $z_{0}$ noktasında bir konform dönüşümdür denir. Eğer $f$ fonksiyonu her $z_{0} \in D$ noktasinda konform ise $f$ fonksiyonuna $D$ bölgesinde konformdur denir. $U$ açı birim diskinde analitik ve $f(0)=f^{\prime}(0)-1=0 \quad$ şartlarını sağlayan $f$ fonksiyonuna ise normalize edilmiş analitik fonksiyon denir. $U$ diskinde analitik normalize edilmiş fonksiyonların sınıfı $\mathcal{A}$ ile gösterilir. Ayrica her $f \in \mathcal{A}$ fonksiyonu

$$
f(z)=z+\sum_{n=2}^{\infty} a_{n} z^{n}
$$

şeklinde Taylor seri açılımına sahiptir. $U$ diskinde normalize edilmiş yalınkat fonksiyonların oluşturduğu sınıfa $\mathcal{S}$ sinıfı denir. $\mathcal{S} \subset \mathcal{A}$ sinıfinın en önemli özelliği bu sınıfa ait her analitik fonksiyon, kompleks düzlemde $U$ birim diskini basit bağlantılı bir bölge üzerine konform ve birebir olarak dönüştürür.

Eğer $f$ fonksiyonu negatif katsayılı yani

$$
f(z)=z-\sum_{n=2}^{\infty}\left|a_{n}\right| z^{n}
$$

ise $T$ ile gösterilir. $\mathrm{Bu}$ sinıf negatif katsayılı fonksiyonlar sinıfı olarak adlandırılır ve $\mathcal{S}$ sinıfinın bir alt sinıfidır (Silverman, 1975).

$\mathcal{S}$ sinıfinın bir diğer önemli alt sinıfı yıldızıl fonksiyonlar sinıfidır. $f \in \mathcal{A}$ olmak üzere $\operatorname{Re}\left(z f^{\prime}(z) / f(z)\right)>\beta \quad(0 \leq \beta<1, z \in U) \quad$ ise $f$ fonksiyonuna $\beta$-mertebeli yıldızıl fonksiyon denir. Bu özellikteki fonksiyonların sınıfı $S T(\beta)$ ile gösterilir. Daha sonra yapılan çalışmalarda yıldızıl fonksiyonların kapsamı genişletilmiştir. Goodman (1991) yaptığı çalışmalarda $f$ fonksiyonunun $U$ açık birim diskinde bulunan $\zeta \in U$ merkezli her dairesel yayı yıldızıl bir yay üzerine resmettiğini göstermiştir. Bu özellikteki bir $f \in \mathcal{A}$ fonksiyonunu düzgün yıldızıl fonksiyon olarak adlandırmıştır. Bu çalışmadan yola çıkarak analitik fonksiyonların birçok alt sınıfı tanımlanmıştır. $\mathrm{Bu}$ alt sınıflardan çalışmamızda kullanılan sınıf Tanım 1.1 ile ifade edilmiştir.

Tanım 1.1. $f$ fonksiyonu (2) formunda tanımlı bir fonksiyon olmak üzere

$$
\operatorname{Re}\left(\frac{f(z)-f(\zeta)}{(z-\zeta) f^{\prime}(z)}\right)>\beta \quad(0 \leq \beta \leq 1,(z, \zeta) \in U \times U)
$$

analitik kriterini sağlıyorsa $\operatorname{USTN}(\beta)$ sinıfındadır denir (Subramanian vd., 1995).

\section{Materyal ve metot}

Poisson, Pascal, Binomial ve Logaritmik gibi negatif olmayan tamsayı değerli dağılım serileri istatistiksel modeller, parametrik olmayan testler, yaşam analizleri gibi birçok alanda önemli kullanımlara sahiptir. En önemli kullanım alanları ise veri analizi olarak da bilinen çok değişkenli veri araştırmalarıdır. Bununla birlikte, günümüzde, bu dağılım serileri Analitik Fonksiyon Teorisi'nde kısmen çalışılmaya başlanmıştır. $\mathrm{Bu}$ alanda literatürdeki ilk çalışma Porwal (2014) tarafından yapılmıştır. Porwal çalışmasında Poisson dağılım fonksiyonu yardımıyla Poisson tipi dağılım serilerini tanımlamıştır. Poisson dağılım fonksiyonu, aritmetik ortalamanın bir ifadesidir. Daha sonra bu serilerin analitik fonksiyonların temel alt sinıflarına ait olup olmadığını ve ait olmas1 durumunda hangi şartlar altında ait olduğunu incelemiştir. Bir başka çalışmada ise ElDeeb vd. (2019) analitik fonksiyonların bazı alt sınıfları için Pascal tipi dağılım serilerini incelemiştir. $\mathrm{Bu}$ çalışmaların ardından, dağılım serileri analitik fonksiyonların diğer alt sınıfları için de araştırılmıştır (Altınkaya ve Yalçın, 2017; Bulboaca ve Murugusundaramoorthy, 2020; Frasin, 2020; Murugusundaramoorthy vd., 2016; Porwal vd., 2020).

$X$ negatif olmayan ayrık rastgele bir değişken, $k$ herhangi bir parametre ve $0 \leq p \leq 1$ olmak üzere Pascal dağılımı

$$
P(X=n)=\left(\begin{array}{c}
n+k-1 \\
k-1
\end{array}\right) p^{n}(1-p)^{k} \quad(n \in\{0,1,2,3, \ldots\})
$$

şeklinde tanımlanır ve bu dağılımdan faydalanarak 
$P(X, z)=z+\sum_{n=2}^{\infty}\left(\begin{array}{c}n+k-2 \\ k-1\end{array}\right) p^{n-1}(1-p)^{k} z^{n}$

formunda kuvvet serisi elde edilir. Elde edilen kuvvet serisi (2) formundaki bir $f$ fonksiyonu için uygulanırsa $P(k, p, z)=2 z-P(X, z)=z-\sum_{n=2}^{\infty}\left(\begin{array}{c}n+k-2 \\ k-1\end{array}\right) p^{n-1}(1-p)^{k} z^{n}(k \geq 1,0 \leq p \leq 1, z \in U)$

bulunur ve $P(k, p, z)$ fonksiyonunun lineer kombinasyonuyla

$$
P_{\sigma}(k, p, z)=(1-\sigma) P(k, p, z)+\sigma z(P(k, p, z))^{\prime}=z-\sum_{n=2}^{\infty}\left(\begin{array}{c}
n+k-2 \\
k-1
\end{array}\right)(1-\sigma+\sigma n) p^{n-1}(1-p)^{k} z^{n}
$$

kuvvet serisi elde edilir. Oran testini kullanarak, yukarıdaki kuvvet serilerinin yakınsaklık yarıçapının sonsuz olduğu söylenebilir.

Lemma 2.1'de bir fonksiyonun Tanım 1.1 ile verilen $\operatorname{USTN}(\beta)$ sınıfına ait olması için sağlaması gereken eşitsizlik verilmiştir.

Lemma 2.1. $\operatorname{USTN}(\beta)$ sinıfina ait (2) formunda tanımlı bir $f$ fonksiyonu

$$
\sum_{n=2}^{\infty}[n(3-\beta)-2]\left|a_{n}\right| \leq 1-\beta
$$

eşitsizliğini sağlar (Subramanian vd., 1995).

Bu çalışmada, dağılım serilerinden Pascal tipi olanlar kullanılarak tanımlanan $P_{\sigma}(k, p, z)$ ve $P(k, p, z)$ fonksiyonlarının $\operatorname{USTN}(\beta)$ analitik fonksiyon sınıfına ait olması için gerekli şartlar elde edilmiştir. Bu amaca ulaşmak için Lemma 2.1 kullanılmıştır.

\section{Bulgular}

Bu bölümde, $P_{\sigma}(k, p, z)$ ve $P(k, p, z)$ fonksiyonlarının $\operatorname{USTN}(\beta)$ analitik fonksiyon sınıfına ait olma şartlarını içeren teoremler ve ispatlar verilmiştir.

Teorem 3.1. $0<p<1$ ve $k \geq 1$ olmak üzere $P_{\sigma}(k, p, z)$ fonksiyonu

$$
\frac{\sigma(3-\beta) k(k+1) p^{2}}{(1-p)^{2}}+\frac{(3-\beta+4 \sigma-2 \sigma \beta) k p}{1-p} \leq(1-\beta)(1-p)^{k}
$$

eşitsizliğini sağlıyorsa $\operatorname{USTN}(\beta)$ sınıfindadır.

İspat. $P_{\sigma}(k, p, z)$ fonksiyonu için Lemma 2.1 ile verilen katsayı eşitsizliği kullanılırsa aşağıdaki eşitsizlik elde edilmelidir.

$$
\sum_{n=2}^{\infty}\left(\begin{array}{c}
n+k-2 \\
k-1
\end{array}\right)(1-\sigma+\sigma n)[n(3-\beta)-2] p^{n-1}(1-p)^{k} \leq 1-\beta .
$$


O halde $P_{\sigma}(k, p, z)$ fonksiyonunun $\operatorname{USTN}(\beta)$ sınıfında olduğunu göstermek için (3) eşitsizliğinin sağlandığını göstermek yeterli olacaktır. Bunun için $n=(n-1)+1, n^{2}=(n-1)(n-2)+3(n-1)+1$ eşitliklerinden faydalanarak

$$
\begin{aligned}
& \sum_{n=2}^{\infty}\left(\begin{array}{c}
n+k-2 \\
k-1
\end{array}\right)(1-\sigma+\sigma n)[n(3-\beta)-2] p^{n-1}(1-p)^{k} \\
& =\sum_{n=2}^{\infty}\left(\begin{array}{c}
n+k-2 \\
k-1
\end{array}\right)[(n-1)(n-2) \sigma(3-\beta)+(n-1)(3-\beta+4 \sigma-2 \sigma \beta)+(1-\beta)] p^{n-1}(1-p)^{k}
\end{aligned}
$$

bulunur. Daha sonra kombinasyon özelliklerinden faydalanmak için

$$
\begin{aligned}
& =\sigma(3-\beta) \sum_{n=2}^{\infty}\left(\begin{array}{c}
n+k-2 \\
k-1
\end{array}\right)(n-1)(n-2) p^{n-1}(1-p)^{k} \\
& +(3-\beta+4 \sigma-2 \sigma \beta) \sum_{n=2}^{\infty}\left(\begin{array}{c}
n+k-2 \\
k-1
\end{array}\right)(n-1) p^{n-1}(1-p)^{k}+(1-\beta) \sum_{n=2}^{\infty}\left(\begin{array}{c}
n+k-2 \\
k-1
\end{array}\right) p^{n-1}(1-p)^{k}
\end{aligned}
$$

eşitliği yazılır. Kombinasyon açılımlarından

$$
\begin{aligned}
& =\sigma(3-\beta) \sum_{n=3}^{\infty}\left(\begin{array}{c}
n+k-2 \\
k+1
\end{array}\right) k(k+1) p^{2} p^{n-3}(1-p)^{k} \\
& +(3-\beta+4 \sigma-2 \sigma \beta) \sum_{n=2}^{\infty}\left(\begin{array}{c}
n+k-2 \\
k
\end{array}\right) k p p^{n-2}(1-p)^{k}+(1-\beta) \sum_{n=2}^{\infty}\left(\begin{array}{c}
n+k-2 \\
k-1
\end{array}\right) p^{n-1}(1-p)^{k} \\
& =\sigma(3-\beta) k(k+1) p^{2} \sum_{n=0}^{\infty}\left(\begin{array}{c}
n+k+1 \\
k+1
\end{array}\right) p^{n}(1-p)^{k}+(3-\beta+4 \sigma-2 \sigma \beta) k p \sum_{n=0}^{\infty}\left(\begin{array}{c}
n+k \\
k
\end{array}\right) p^{n}(1-p)^{k} \\
& +(1-\beta)(1-p)^{k} \sum_{n=0}^{\infty}\left(\begin{array}{c}
n+k-1 \\
k-1
\end{array}\right) p^{n}-(1-\beta)(1-p)^{k}
\end{aligned}
$$

elde edilir. Elde edilen bu son eşitlikte $\sum_{n=0}^{\infty}\left(\begin{array}{c}n+k-1 \\ k-1\end{array}\right) p^{n}=\frac{1}{(1-p)^{k}}$ olduğu kullanılırsa

$$
=\frac{\sigma(3-\beta) k(k+1) p^{2}}{(1-p)^{2}}+\frac{(3-\beta+4 \sigma-2 \sigma \beta) k p}{1-p}+(1-\beta)-(1-\beta)(1-p)^{k} \leq 1-\beta .
$$

bulunur. Bu da ispatı tamamlar.

Teorem 3.2. $0<p<1$ ve $k \geq 1$ olmak üzere $P(k, p, z)$ fonksiyonu

$$
\frac{(3-\beta) k p}{1-p} \leq(1-\beta)(1-p)^{k}
$$

eşitsizliğini sağlıyorsa $\operatorname{USTN}(\beta)$ sınıfındadır. 
İspat. $P(k, p, z)$ fonksiyonu için Lemma 2.1 ile verilen katsayı eşitsizliği kullanılarak (4) eşitsizliği elde edilmelidir.

$\sum_{n=2}^{\infty}\left(\begin{array}{c}n+k-2 \\ k-1\end{array}\right)[n(3-\beta)-2] p^{n-1}(1-p)^{k} \leq 1-\beta$

bulunur. Teorem 3.1'de kullanılan benzer işlemlerle

$$
\begin{aligned}
& \sum_{n=2}^{\infty}\left(\begin{array}{c}
n+k-2 \\
k-1
\end{array}\right)[n(3-\beta)-2] p^{n-1}(1-p)^{k}=\sum_{n=2}^{\infty}\left(\begin{array}{c}
n+k-2 \\
k-1
\end{array}\right)[(n-1)(3-\beta)+(1-\beta)] p^{n-1}(1-p)^{k} \\
& =(3-\beta) \sum_{n=2}^{\infty}\left(\begin{array}{c}
n+k-2 \\
k
\end{array}\right) k p p^{n-2}(1-p)^{k}+(1-\beta) \sum_{n=2}^{\infty}\left(\begin{array}{c}
n+k-2 \\
k-1
\end{array}\right) p^{n-1}(1-p)^{k} \\
& =(3-\beta) k p \sum_{n=0}^{\infty}\left(\begin{array}{c}
n+k \\
k
\end{array}\right) p^{n}(1-p)^{k}+(1-\beta) \sum_{n=0}^{\infty}\left(\begin{array}{c}
n+k-1 \\
k-1
\end{array}\right) p^{n}(1-p)^{k}-(1-\beta)(1-p)^{k} \\
& =\frac{(3-\beta) k p}{1-p}+(1-\beta)-(1-\beta)(1-p)^{k} \leq 1-\beta
\end{aligned}
$$

istenilen eşitsizlik elde edilir.

\section{Tartışma ve sonuçlar}

$\mathrm{Bu}$ çalışmada Pascal tipi dağılım serilerinden faydalanarak tanımlanan $P_{\sigma}(k, p, z)$ ve $P(k, p, z)$ fonksiyonlarının $\operatorname{USTN}(\beta)$ analitik fonksiyon sınıfına ait olması için gerekli şartlar elde edilmiştir. Bu yaklaşım analitik fonksiyonların diğer sınıflarına genişletilebilir. Aynı zamanda dağ 1 lım serilerinden faydalanarak yeni fonksiyonlar tanımlanabilir ve benzer özellikleri incelenebilir.

Bilimin ve teknolojinin ilerleyen her aşamasında kendisine uygulama alanı bulabilen dağılım serileri Kompleks analizde oldukça önemli olan analitik fonksiyonlar teorisine de yenilikler getirebilir.

\section{Kaynaklar}

Altınkaya, Ş. and Yalçın, S. (2017). Poisson distribution series for certain subclasses of starlike functions with negative coefficients. Annals of Oradea Universty Mathematics Fascicola, 24(2), 5-8.

Bulboaca, T. and Murugusundaramoorthy, G. (2020). Univalent functions with positive coefficients involving Pascal distribution series. Communications of the Korean Mathematical Society, 35(3), 867-877. https://doi.org/10.4134/CKMS.c190413
El-Deeb, S., Bulboaca, T. and Dziok, J. (2019). Pascal distribution series connected with certain subclasses of univalent functions. Kyungpook Mathematical Journal, 59(2), 301-314. https://doi.org/10.5666/KMJ.2019.59.2.301

Frasin, B. (2020). Subclasses of analytic functions associated with Pascal distribution series. Advances in the Theory of Nonlinear Analysis and its Applications, 4(2), 92-99. https://doi.org/10.31197/atnaa.692948

Goodman, A. (1991). On uniformly starlike functions. Journal of Mathematical Analysis and Applications, 155(2), 364-370.

Murugusundaramoorthy, G., Vijaya, K. and Porwal, S. (2016). Some inclusion results of certain subclass of analytic functions associated with Poisson distribution series. Hacettepe Journal of Mathematics and Statistics, 45(4), 1101-1107.

Porwal, S. (2014). An application of a Poisson distribution series on certain analytic functions. Journal of Complex Analysis, 2014, 1-3. http://dx.doi.org/10.1155/2014/984135

Porwal, S., Magesh, N. and Abirami, C. (2020). Certain subclasses of analytic functions associated with Mittag-Lefflertype Poisson distribution series. Boletín de la Sociedad Matemática Mexicana, 26(3), 1035-104. https://doi.org/10.1007/s40590-020-00288-x 
Silverman, H. (1975). Univalent functions with negative coefficients. Proceedings of the American Mathematical Society, 51(1), 109-116.
Subramanian, K., Murugusundaramoorthy, G., Balasubrahmanyam, P. and Silverman, $H$. (1995). Subclasses of uniformly convex and uniformly starlike functions. Mathematica Japonicae, 42(3), 517-522. 